\title{
Spectrum Clouds: A Session Based Spectrum Trading System for Multi-hop Cognitive Radio Networks
}

\author{
Miao Pan*, Pan $\mathrm{Li}^{\dagger}$, Yang Song ${ }^{\ddagger}$, Yuguang Fang* and Phone Lin ${ }^{\S}$ \\ ${ }^{*}$ Department of Electrical and Computer Engineering, University of Florida, Gainesville, FL 32611 \\ ${ }^{\dagger}$ Department of Electrical and Computer Engineering, Mississippi State University, Mississippi State, MS 39762 \\ ${ }^{\ddagger}$ IBM T. J. Watson Research Center, Hawthorne, NY 10532 \\ $\S$ Department of Computer Science and Information Engineering, National Taiwan University, Taipei, Taiwan.
}

\begin{abstract}
Spectrum trading creates more accessing opportunities for secondary users (SUs) and economically benefits the primary users (PUs). However, it is challenging to implement spectrum trading in multi-hop cognitive radio networks (CRNs) due to harsh cognitive radio (CR) requirements on SUs' devices and complex conflict and competition relationship among different CR sessions. Unlike the per-user based spectrum trading designs in previous studies, in this paper, we propose a novel session based spectrum trading system, spectrum clouds, in multihop CRNs. In spectrum clouds, we introduce a new service provider, called secondary service provider (SSP), to harvest the available spectrum bands and facilitate the accessing of SUs without CR capability. The SSP also conducts spectrum trading among CR sessions w.r.t. their conflicts and competitions. Leveraging a 3-dimensional (3-D) conflict graph, we mathematically describe the conflicts and competitions among the candidate sessions for spectrum trading. Given the rate requirements and bidding values of candidate trading sessions, we formulate the optimal spectrum trading into the SSP's revenue maximization problem under multiple cross-layer constraints in multi-hop CRNs. In view of the NP-hardness of the problem, we have also developed heuristic algorithms to pursue feasible solutions. Through extensive simulations, we show that the solutions found by the proposed algorithms are close to the optimal one.
\end{abstract}

\section{INTRODUCTION}

Nowadays, more and more people, families and companies rely on wireless services for their daily life and business, which leads to a booming growth of various wireless networks and a dramatic increase in the demand for radio spectrum. In parallel with that, current static spectrum allocation policy of Federal Communications Commission (FCC) [1]-[3] results in the exhaustion of available spectrum, while a lot of licensed spectrum bands are extremely under-utilized. Experimental tests in academia [4], [5] and measurements conducted in industries [6], [7] both show that even in the most crowed region of big cities (e.g., Washington, DC, Chicago, New York City, etc.), many licensed spectrum bands are not used in certain geographical areas and are idle most of the time. Those studies spur the FCC to open up licensed spectrum bands and pursue new innovative technologies to encourage dynamic use of the under-utilized spectrum [1]. As one of

This work was partially supported by the U.S. National Science Foundation under grants CNS-1147813/1147851 and ECCS-1129062/1128768. The work of Y. Fang was also partially supported by the National Natural Science Foundation of China under grant 61003300 and the China 111 Project under grant B08038. The work of P. Lin was partially supported by National Science Council, National Taiwan University and Intel Corporation under Grants NSC 100-2911-I-002-001, and 10R70501. the most promising solutions, cognitive radio (CR) technology releases the spectrum from shackles of authorized licenses, and enables secondary users (SUs) to opportunistically access to the vacant licensed spectrum bands in either temporal or spatial domain.

The idea of opportunistic using licensed spectrum bands has initiated the spectrum trading in multi-hop cognitive radio networks (CRNs) and promoted a lot of interesting research on the design of spectrum trading systems [8][12]. Through spectrum trading, primary users (PUs) can sell/lease/auction their vacant spectrum for monetary gains, and SUs can purchase/rent/bid the available licensed spectrum if they suffer from the lack of radio resources to support their traffic demands. However, to trade the licensed spectrum and opportunistically access to these bands, SUs' handsets have to be frequency-agile [2], [13]. It is imperative for the SUs' devices to have the CR capability such as exploring licensed spectrum bands, reconfiguring RF, switching frequencies across a wide spectrum range (i.e., from $20 \mathrm{MHz}$ to $2.5 \mathrm{GHz}[13]-[15])$, sending and receiving packets over non-contiguous spectrum bands, etc. Although some of the desired features may be realized in future, enormous amount of time and efforts must be spent in hardware designs and signal processing in order to implement these features in light weight radios [2], [13], [14]. In addition, to attract customers for any new technologies, there is no reason to enforce the users to replace their communication devices or to increase the complexity on the customers' side. For spectrum trading in CRNs, it is always appreciated to minimize the changes on the handsets of SUs while facilitating the spectrum trading to maximize the spectral efficiency.

Except for the harsh requirements on SUs' devices, another primary challenge for spectrum trading in multi-hop CRNs is how SUs conduct the multi-hop CR communications using the purchased spectrum. Most existing work focuses on peruser based spectrum trading [9]-[12], i.e., each SU purchases available bands from PUs and uses the purchased spectrum for communications. Unfortunately, those spectrum trading designs are confronted with several critical problems when they are deployed in multi-hop CRNs. For instance, it is not clear whom a SU communicates with (i.e., the destination $\mathrm{SU}$ or the receiver is not explicitly specified); it is not clear how to find a common band between two SUs to establish communications; it is not clear what kind of quality of service (e.g., throughput, delay, rate or bandwidth requirement, etc.) 
can be supported. Besides, although some of prior spectrum trading systems consider the impact of frequency reuse [9][12], [16], they ignore almost all the other factors, such as interference mitigation, link scheduling, flow routing, etc., which may significantly affect the performance of CR sessions in multi-hop CRNs.

To address the challenges above, in this paper, we propose a session based spectrum trading system, spectrum clouds, for multi-hop CRNs. In order to facilitate the spectrum trading of SUs without CR capability, a novel network architecture and new network entities are introduced in spectrum clouds. Under the proposed architecture of CRNs, we study the session based spectrum trading instead of per-user based spectrum trading. Given the rate requirements and bidding values ${ }^{1}$ of candidate CR sessions, we endeavor to conduct the optimal spectrum trading under multiple constraints (e.g., the availability of spectrum bands, the competition among different CR sessions, link scheduling constraints, flow routing constraints, etc.) in multi-hop CRNs. We mathematically formulate these concerns into an optimization problem and provide both the nearoptimal solution and the feasible solution in this work. Our salient contributions are listed as follows.

- Different from the architecture of traditional spectrum trading systems, we introduce a new emerging service provider, called Secondary Service Provider (SSP), in spectrum clouds, and assume the SSP has already established some partial infrastructure with CR mesh routers ${ }^{2}$ at low cost to provide coverage in the area of interest. Suppose that the SSP has its own bands (i.e., basic bands) and can harvest the available licensed spectrum bands. To facilitate the accessing of SUs without CR devices, all the $\mathrm{CR}$ mesh routers are equipped with multiple CR radios. Under the guidance of the SSP, SUs access their nearby CR mesh routers using basic bands and deliver packets via $C R$ mesh routers using both basic bands and harvested bands. Given rate requirements and bidding values of $\mathrm{CR}$ sessions with different source/destination SUs, the SSP seeks to optimally trade the spectrum with a objective of maximizing his revenue under multiple constraints in multi-hop CRNs, i.e., the spectrum availability, link scheduling and flow routing constraints.

- Similar to the multi-dimensional conflict graph illustrated in [17], we employ a 3-dimensional (3-D) conflict graph to characterize the conflict relations among CR links in spectrum clouds. Based on the 3-D conflict graph, we mathematically describe the competition among CR sessions for radio spectrum as well as the link scheduling and routing constraints. Furthermore, we formulate the optimal session based spectrum trading into the SSP's revenue maximization problem under those cross-layer constraints. Given all the independent sets in CRNs, we can relax the integer variables in the formulation, solve the optimization problem by linear programming, and

\footnotetext{
${ }^{1}$ In this paper, bidding values generally represent how much the SUs are willing to pay for purchasing/renting/bidding for the available spectrum, which can be used for the traffic delivery of corresponding CR sessions.

${ }^{2}$ In the rest of this paper, we use the words CR router/CR mesh router/router interchangeably.
}

find the upper bound of the SSP's revenue for session based spectrum trading in multi-hop CRNs.

- Since the competition relationship between any two sessions is represented by binary values, it is NP-hard to solve the formulated optimization, in which these integer constraints are involved [18], [19]. To pursue feasible solutions, we develop a heuristic relax-and-fix algorithm to determine the values of integer variables. Briefly speaking, we divide all the $\mathrm{CR}$ sessions into different sets and relax-and-fix the integer variables for $\mathrm{CR}$ sessions in one session set after another. If there exists a feasible solution, it yields a lower bound to the original optimization problem.

- By carrying out extensive simulations in both grid topology and random topology, we demonstrate that the proposed session based spectrum trading system has great advantages over the per-user based ones in multi-hop CRNs. We also compare the upper bound and lower bound determined by the heuristic algorithm at different data sets, and show that the feasible solutions obtained by the proposed algorithm are really close to the optimal one in terms of the SSP's revenue.

The rest of the paper is organized as follows. In Section II, we introduce the system architecture of spectrum clouds, corresponding network settings and related models in multi-hop CRNs. In Section III, we mathematically describe link scheduling and routing constraints in spectrum clouds, formulate the session based spectrum trading under multiple constraints into an optimization problem and near-optimally solve it by linear programming. In Section IV, we develop the heuristic algorithms for feasible solutions. Finally, we conduct simulations and analyze the performance results in Section V, and draw concluding remarks in Section VI.

\section{NeTWORK MODEL}

\section{A. System Architecture for Spectrum Clouds}

We consider the proposed spectrum trading system in multihop CRNs, spectrum clouds, consisting of the SSP, a group of SUs, a set of CR mesh routers and a collection of available licensed spectrum bands ${ }^{3}$ with unequal size of bandwidths as shown in Fig. 1(a). The SSP is an independent wireless service provider (e.g., a base station or an access point) with its own spectrum, i.e., the SSP's basic bands (potentially congested already), and is able to collectively harvest the available licensed bands. The SSP has also deployed some CR mesh routers at low cost to facilitate the accessing of SUs. SUs are just end-users not subscribed to primary services. No specific requirements are imposed on the SUs' communication devices. They could be any devices using any accessing technologies (e.g. laptops or desktop computers using Wi-Fi, cell phones using GSM/GPRS, smart phones using 3G/4G/NxtG accessing technology, etc.). SUs can access to the basic bands owned by the SSP, but they cannot be tuned to the harvested licensed

\footnotetext{
${ }^{3}$ Taking the least-utilized spectrum bands introduced in [20] for example, we found that the bandwidth between $[1240,1300] \mathrm{MHz}$ (allocated to amateur radio) is $60 \mathrm{MHz}$, while bandwidth between [1525, 1710] $\mathrm{MHz}$ (allocated to mobile satellites, GPS systems, and meteorological applications) is $185 \mathrm{MHz}$.
} 
frequency. The CR mesh routers deployed by the SSP have CR capability and are equipped with multiple CR radios.

Under spectrum clouds' architecture, the mobile SUs report their online traffic requests, which include source/destination, rate requirements and corresponding bidding values of the SUs' sessions, to their nearby CR mesh routers via basic bands. The fixed CR mesh routers collect these requests from different end-users and report them to the SSP. Depending on the bidding values, rate requirements and the available spectrum resources, the SSP makes decisions on the accessing/denial of the SUs' sessions, and jointly conducts link scheduling and flow routing among CR mesh routers for SUs' traffic delivery. Following the guidance of the SSP, the CR mesh routers form unicast CR communication sessions and deliver packets using both the leftover basic bands and harvested bands as shown in Fig. 1(a).

In traditional spectrum trading systems, the spectrum bands to sell/lease/auction are known to every SU. Due to broadcasting nature of wireless transmissions, the SU may also know his potential competitors and overhear their bids, so that many schemes are proposed to ensure that the spectrum trading is not manipulated in multi-hop CRNs [9], [12]. By contrast, in spectrum clouds, the SU has no idea about the specific spectrum allocation across the whole session (i.e., from the source to the destination). Even if a SU overhears the bids of other SUs, it is not helpful since the SU is not sure who are his competitors for spectrum usage. Besides, spectrum clouds can support session based spectrum trading in multihop CRNs, whereas the other systems can only support singlehop spectrum trading as shown in Fig. 1(b).

\section{B. Network Configuration}

Suppose there are $\mathcal{N}=\{1,2, \cdots, n, \cdots, N\}$ CR mesh routers, each $\mathrm{CR}$ mesh router has $\mathcal{H}=\{1,2, \cdots, h, \cdots, H\}$ radio interfaces, and these $\mathrm{CR}$ mesh routers form a set of $\mathcal{L}$ unicast communication sessions according to SUs' requests. Each session has a rate requirement and a corresponding bidding value. Denote the source/destination CR router of session $l \in \mathcal{L}=\{1,2, \cdots, l, \cdots, L\}$ by $s_{r}(l) / d_{t}(l)$, and let $(r(l), b(l))$ be the rate requirement-bidding value pair for session $l \in \mathcal{L}$. Assume the SUs' usage of basic bands in the multi-hop CRNs is a priori information. The CR routers are able to use the rest of basic spectrum owned by the SSP. The CR routers are also allowed to communicate with each other by opportunistically accessing to the licensed bands when the primary services are not active, but they must evacuate from these bands immediately when primary services become active. Considering the geographical location of the $\mathrm{CR}$ routers, the available spectrum bands at one $\mathrm{CR}$ router may be different from another one in the network. To put it in a mathematical way, let $\mathcal{M}=\{1,2, \cdots, m \cdots, M\}$ be the band set including the available basic bands and licensed bands with different bandwidths $\mathcal{W}=\left\{W^{1}, W^{2}, \cdots, W^{m}, \cdots, W^{M}\right\}$ for communications, and $\mathcal{M}_{i} \subseteq \mathcal{M}$ represent the set of available bands at $\mathrm{CR}$ router $i \in \mathcal{N} . \mathcal{M}_{i}$ may be different from $\mathcal{M}_{j}$, where $j$ is not equal to $i$, and $j \in \mathcal{N}$, i.e., possibly $\mathcal{M}_{i} \neq \mathcal{M}_{j}$.

C. Other Related Models in Multi-hop CRNs

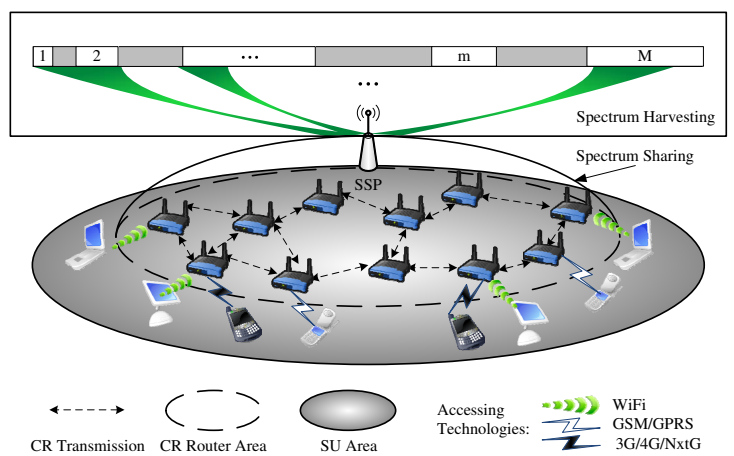

(a) System architecture for spectrum clouds.

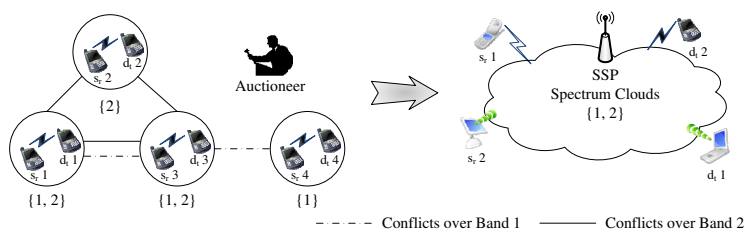

(b) A schematic for comparison between traditional spectrum trading mechanisms and spectrum clouds.

Fig. 1. A novel architecture for spectrum trading in multi-hop CRNs.

1) Transmission Range and Interference Range: Suppose all CR mesh routers use the same power $P$ for transmission. The power propagation gain [20], [21] is

$$
g_{i j}=\gamma \cdot d_{i j}^{-\alpha},
$$

where $\alpha$ is the path loss factor, $\gamma$ is an antenna related constant, and $d_{i j}$ is the distance between CR routers $i$ and $j$. We assume that the data transmission is successful only if the received power at the receiver exceeds the receiver sensitivity, i.e., a threshold $P_{T}$. Meanwhile, we assume interference becomes non-negligible only if it is over a threshold of $P_{I}$ at the receiver. Thus, the transmission range for a $\mathrm{CR}$ router is $R_{T}=\left(\gamma P / P_{T}\right)^{1 / \alpha}$, which comes from $\gamma \cdot\left(R_{T}\right)^{-\alpha} \cdot P=P_{T}$. Similarly, based on the interference threshold $P_{I}\left(P_{I}<P_{T}\right)$, the interference range for a CR router is $R_{I}=\left(\gamma P / P_{I}\right)^{1 / \alpha}$. It is obvious that $R_{I}>R_{T}$ since $P_{I}<P_{T}$.

In the widely used protocol model [17], [18], [20], [22][24], the interference range is typically 2 or 3 times of the transmission range, i.e., $\frac{R_{I}}{R_{T}}=2$ or 3 . These two ranges may vary with frequency. The conflict relationship between two links over the same frequency band can be determined by the specified interference range. In addition, if the interference range is properly set, the protocol model can be accurately transformed into the physical model as illustrated in [25].

2) Link Capacity/Achievable Data Rate: According to Shannon-Hartley theorem, if CR router $i$ sends data to CR router $j$ on link $(i, j)$ with band $m$, the capacity of link $(i, j)$ with band $m$ is

$$
c_{i j}^{m}=W^{m} \log _{2}\left(1+\frac{g_{i j} P}{\eta}\right),
$$

where $\eta$ is the ambient Gaussian noise power at CR mesh router $j^{4}$. Depending on different modulation schemes, the

\footnotetext{
${ }^{4}$ Note that the denominator inside the $\log$ function contains only $\eta$. This is because of one of our interference constraints, i.e., when CR router $i$ is transmitting to CR router $j$ on band $m$, then all the other neighbors of router $j$ within its interference range are prohibited from using this band. We will address the interference constraints in details in the following section.
} 
achievable data rate is actually determined by the SNR at the receiver and receiver sensitivity [24], [26]. However, in most of existing literature [17], [18], [20], the achievable data rate is approximated by (2), even though this data rate can never be achieved in practical. In this paper, we follow the same approximation. Note that this approximation will not affect the theoretical analysis or performance comparison in this work.

\section{Optimal SPECTRUM TRADING UNDER CROSS-LAYER CONSTRAints In MULTI-HOP CRNS}

We exploit binary value $\delta(l)$ to denote the success/failure of spectrum trading for session $l$, i.e.,

$$
\delta(l)= \begin{cases}1, & \text { session } l \text { is accessed by the SSP } \\ 0, & \text { session } l \text { is denied by the SSP. }\end{cases}
$$

To make the decision of accessing/denying a session $l \in \mathcal{L}$, the SSP must consider both the rate requirement and bidding value of session $l$. Besides, to effectively utilize the leftover basic spectrum and the harvested licensed spectrum, it is necessary for the SSP to schedule data transmission among different CR mesh routers under joint spectrum assignment, link scheduling and flow routing constraints. In the rest of this section, we first extend the conflict graph [24], [26] to characterize the interference relationship among CR links in spectrum clouds. Then, based on the extended conflict graph, we mathematically describe link scheduling and flow routing constraints and formulate the spectrum trading into the revenue maximization problem of the SSP under multiple constraints. By relaxing the integral variables, we solve the optimization problem and provide an upper-bound of the SSP's revenue.

\section{A. Extended Conflict Graph, Cliques and Independent Sets}

1) Construction of 3-Dimensional (3-D) Conflict Graph: Regarding the availability of spectrum bands and radios at CR mesh routers, we introduce a 3-D conflict graph to characterize the interference relationship among CR links in spectrum clouds. Following the definitions in [17], we interpret a $\mathrm{CRN}$ as a three-dimensional resource space, with dimensions defined by links, the set of available bands and the set of available radios. In a 3 -D conflict graph $\mathcal{G}(\mathcal{V}, \mathcal{E})$, each vertex corresponds to a link-band-radio (LBR) tuple, i.e.,

$$
\text { link-band-radio: } \quad((i, j), m,(u, v)),
$$

where $i \in \mathcal{N}, m \in \mathcal{M}_{i} \bigcap \mathcal{M}_{j}, j \in \mathcal{T}_{i}^{m}, u \in \mathcal{H}_{i}$ and $v \in \mathcal{H}_{j}$. Here, $\mathcal{T}_{i}^{m}$ is the set of $\mathrm{CR}$ mesh routers within $\mathrm{CR}$ router $i$ 's transmission range. The LBR tuple indicates that the CR router $i$ transmits data to $\mathrm{CR}$ router $j$ on band $m$, where radio interfaces $u$ and $v$ are used at sending CR router and receiving $\mathrm{CR}$ router, respectively. Based on the definition of LBR tuples, we can enumerate all combinations of $\mathrm{CR}$ mesh routers, the vacant bands and the available radios, which can potentially enable CR communication links.

Different from multi-channel multi-radio networks [17], the availability of bands and radios (i.e., the leftover radios after collecting SUs' traffic) at each CR router in CRNs may be different, i.e., for $i, j \in \mathcal{N}$, maybe $\mathcal{M}_{i} \neq \mathcal{M}_{j}$ and $\mathcal{H}_{i} \neq \mathcal{H}_{j}$. Similar to the interference conditions in [17], [18], [20], two LBR tuples are defined to interfere with each other if either

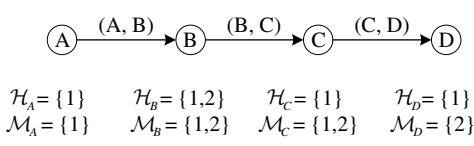

(a) Toy topology in spectrum clouds.

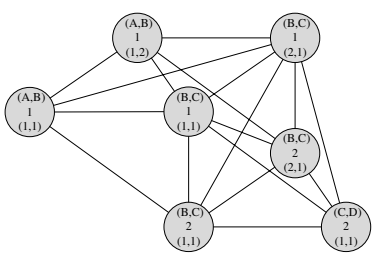

(b) 3-D conflict graph.
Fig. 2. Conflict relationship represented by 3-D conflict graph in CRNs.

of the following conditions is true: (i) if two different LBR tuples are using the same band, the receiving $\mathrm{CR}$ router of one tuple is in the interference range of the transmitting $\mathrm{CR}$ router in the other tuple; (ii) two different LBR tuples have the same radios at one or two CR routers.

Note that the first condition not only represents co-band interference but also inherently covers the following two cases: any $\mathrm{CR}$ router cannot transmit to multiple routers on the same band; any CR router cannot use the same band for concurrent transmission and reception, due to "self-interference" at the physical layer. Meanwhile, the second condition represents the radio interface conflicts, i.e., a single radio cannot support multiple transmissions (either transmitting or receiving) simultaneously. According to these conditions, we connect two vertices in $\mathcal{V}$ with an undirected edge in $\mathcal{G}(\mathcal{V}, \mathcal{E})$, if their corresponding LBR tuples interfere with each other.

For illustrative purposes, we take a simple example to show how to construct a 3-D conflict graph. In this toy CRNs, we assume there are four CR routers with CR transceivers, i.e., $A, B, C$ and $D$, and two bands, i.e., band 1 and band 2. Depending on the geographic locations, the set of currently available bands and radios at one CR router may be different from that at another CR router as shown in Fig. 2(a). For example, the currently available band and radio sets for $A$ are $\mathcal{M}_{A}=\{1\}$ and $\mathcal{H}_{A}=\{1\}$, and the band and radio sets for $B$ are $\mathcal{M}_{B}=\{1,2\}$ and $\mathcal{H}_{B}=\{1,2\}$. Furthermore, we use $d(\cdot)$ to represent Euclidean distance and suppose that $d(A, B)=d(B, C)=d(C, D)=d(D, E)$ $=R_{T}=0.5 R_{I}$. Given the above assumptions, we can establish the corresponding 3-D conflict graph as depicted in Fig. 2(b). Here, each vertex corresponds to an LBR tuple, for example, vertex $((A, B), 1,(1,1))$ corresponds to LBR tuple $((A, B), 1,(1,1))$. Note that there is edge between vertices $((A, B), 1,(1,1))$ and $((B, C), 1,(2,1))$ because $(A, B)$ is incident to $(B, C)$ over band 1 . There is an edge between vertices $((A, B), 1,(1,1))$ and $((B, C), 2,(1,1))$ because they share a radio in common at $\mathrm{CR}$ router $B$. Similar analysis applies to the other vertices in the conflict graph as well.

2) 3-D Independent Sets and Conflict Cliques: Given a 3D conflict graph $\mathcal{G}=(\mathcal{V}, \mathcal{E})$ representing spectrum clouds, we describe the impact of vertex $i \in \mathcal{V}$ on vertex $j \in \mathcal{V}$ as follows,

$w_{i j}=\left\{\begin{array}{l}1, \text { if there is an edge between vertex } i \text { and } j \\ 0, \text { if there is no edge between vertex } i \text { and } j,\end{array}\right.$

where two vertices correspond to two LBR tuples, respectively.

Provided that there is a vertex set $\mathcal{I} \subseteq \mathcal{V}$ and an LBR tuple $i \in \mathcal{I}$ satisfying $\sum_{j \in \mathcal{I}, i \neq j} w_{i j}<1$, the transmission at LBR tuple $i$ will be successful even if all the other LBR tuples in the 
set $\mathcal{I}$ are transmitting at the same time. If any $i \in \mathcal{I}$ satisfies the condition above, we can schedule the transmissions over all these LBR tuples in $\mathcal{I}$ to be active simultaneously. Such a vertex/LBR tuple set $\mathcal{I}$ is called a $3-\mathrm{D}$ independent set. If adding any one more LBR tuple into a 3-D independent set $\mathcal{I}$ results in a non-independent one, $\mathcal{I}$ is defined as a maximum 3$\mathrm{D}$ independent set. Besides, if there exists a vertex/LBR tuple set $\mathcal{Z} \subseteq \mathcal{V}$ and any two vertexes $i$ and $j$ in $\mathcal{Z}$ satisfying $w_{i j} \neq 0$ (i.e., LBR tuples $i$ and $j$ cannot be scheduled to transmit successfully at the same time.), $\mathcal{Z}$ is called a $3-\mathrm{D}$ conflict clique. If $\mathcal{Z}$ is no longer a 3-D conflict clique after adding any one more LBR tuple, $\mathcal{Z}$ is defined as a maximum 3-D conflict clique.

\section{B. CR Link Scheduling and Flow Routing Constraints}

1) CR Link Scheduling Constraints: Link scheduling can be conducted in time domain, in frequency domain or in both of them [18], [20]. In this paper, we only focus on time based link scheduling.

Given the 3-D conflict graph $\mathcal{G}=(\mathcal{V}, \mathcal{E})$ constructed from the spectrum clouds, suppose we can list all maximum 3-D independent sets ${ }^{5}$ as $\mathscr{I}=\left\{\mathcal{I}_{1}, \mathcal{I}_{2}, \cdots, \mathcal{I}_{q}, \cdots, \mathcal{I}_{Q}\right\}$, where $Q$ is $|\mathscr{I}|$, and $\mathcal{I}_{q} \subseteq \mathcal{V}$ for $1 \leq q \leq Q$. At any time, at most one maximum 3-D independent set can be active to transmit packets for all LBR tuples in that set. Let $\lambda_{q} \geq 0$ denote the time share scheduled to the maximum 3-D independent set $\mathcal{I}_{q}$, and

$$
\sum_{1 \leq q \leq Q} \lambda_{q} \leq 1, \quad \lambda_{q} \geq 0(1 \leq q \leq Q) .
$$

Let $r_{i j}^{m}\left(\mathcal{I}_{q}\right)$ be the data rate for CR link $(i, j)$ over band $m$, where $r_{i j}^{m}\left(\mathcal{I}_{q}\right)=0$ if LBR tuple $((i, j), m,(u, v)) \notin \mathcal{I}_{q}$; otherwise, $r_{i j}^{m}\left(\mathcal{I}_{q}\right)$ is the achievable data rate for CR link $(i, j)$ over band $m$, which can be calculated from (2). Therefore, by exploiting the $3-\mathrm{D}$ maximum independent set $\mathcal{I}_{q}$, the flow rate that link $(i, j)$ can support over band $m$ in the time share $\lambda_{q}$ is $\lambda_{q} r_{i j}^{m}\left(\mathcal{I}_{q}\right)$. Let $f_{i j}(l)$ represent the flow rate of the session $l$ over link $(i, j)$, where $i \in \mathcal{N}, l \in \mathcal{L}$ and $j \in \bigcup_{m \in \mathcal{M}_{i}} \mathcal{T}_{i}^{m}$. Then, the trading CR sessions are feasible at link $(i, j)$ if there exists a schedule of the maximum 3-D independent sets satisfying

$$
\sum_{l \in \mathcal{L}}^{s_{r}(l) \neq j, d_{t}(l) \neq i} f_{i j}(l) \delta(l) \leq \sum_{q=1}^{|\mathscr{I}|} \lambda_{q} \sum_{m \in \mathcal{M}_{i} \cap \mathcal{M}_{j}} r_{i j}^{m}\left(\mathcal{I}_{q}\right) .
$$

2) CR Routing Constraints: As for routing, the SSP will help the source $\mathrm{CR}$ mesh router to find the available paths and employ a number of relay $\mathrm{CR}$ mesh routers to forward the data packets toward its destination $\mathrm{CR}$ mesh router. It is obvious that there should be more than one path involved in data delivery since multi-path routing ${ }^{6}$ is more flexible to route the traffic from a source router to its destination. Similar to the modeling in [18], [20], we mathematically present routing constraints as follows.

${ }^{5}$ It is a NP-complete problem to find all maximum independent sets in $\mathcal{G}$ [17], [27], [28], which will be further addressed later in this paper. In this subsection, we make the assumption we could find all the maximum independent sets just for the convenience of our theoretical analysis.

${ }^{6}$ The multiple radios of $\mathrm{CR}$ routers allow for multi-path routing.
To simplify the notation, let $\mathcal{T}_{i}=\bigcup_{m \in \mathcal{M}_{i}} \mathcal{T}_{i}^{m}$. If CR mesh router $i$ is the source router of session $l$, i.e., $i=s_{r}(l)$, then

$$
\begin{gathered}
\sum_{j \in \mathcal{T}_{i}} f_{j i}(l)=0 . \\
\sum_{j \in \mathcal{T}_{i}} f_{i j}(l) \delta(l)=r(l) \delta(l),
\end{gathered}
$$

where $\delta(l) \in\{0,1\}$ indicates whether session $l$ is accepted by the SSP (i.e., session $l$ wins the opportunity for data transmission via spectrum trading) or not.

If $\mathrm{CR}$ mesh router $i$ is an intermediate relay router of session $l$, i.e., $i \neq s_{r}(l)$ and $i \neq d_{t}(l)$, then

$$
\sum_{j \in \mathcal{T}_{i}}^{j \neq s_{r}(l)} f_{i j}(l) \delta(l)=\sum_{p \in \mathcal{T}_{i}}^{p \neq d_{t}(l)} f_{p i}(l) \delta(l)
$$

If CR mesh router $i$ is the destination router of session $l$, i.e., $i=d_{t}(l)$, then

$$
\sum_{j \in \mathcal{T}_{i}} f_{j i}(l) \delta(l)=r(l) \delta(l) .
$$

Note that if (7), (8) and (9) are satisfied, it can be easily verified that (10) must be satisfied. As a result, it is sufficient to list only (7), (8) and (9) as CR routing constraints in spectrum clouds.

\section{Optimal Spectrum Trading under Multiple Constraints}

With the proposed trading system, spectrum clouds, the optimal spectrum trading problem under multiple constraints in multi-hop CRNs can be formulated as follows.

$$
\begin{aligned}
& \text { Maximize } \sum_{l \in \mathcal{L}} b(l) \delta(l) \\
& \text { s.t.: } \quad \sum_{j \in \mathcal{T}_{i}} f_{j i}(l)=0 \quad\left(l \in \mathcal{L}, i=s_{r}(l)\right) \\
& \sum_{j \in \mathcal{T}_{i}} f_{i j}(l) \delta(l)=r(l) \delta(l) \quad\left(l \in \mathcal{L}, i=s_{r}(l)\right) \\
& \sum_{j \in \mathcal{T}_{i}}^{j \neq s_{r}(l)} f_{i j}(l) \delta(l)=\sum_{p \in \mathcal{T}_{i}}^{p \neq d_{t}(l)} f_{p i}(l) \delta(l) \\
& \left(l \in \mathcal{L}, i \in \mathcal{N}, i \neq s_{r}(l), d_{t}(l)\right) \\
& \sum_{l \in \mathcal{L}}^{s_{r}(l) \neq j, d_{t}(l) \neq i} f_{i j}(l) \delta(l) \leq \sum_{q=1}^{|\mathscr{I}|} \lambda_{q} \sum_{m \in \mathcal{M}_{i} \cap \mathcal{M}_{j}} r_{i j}^{m}\left(\mathcal{I}_{q}\right) \\
& \left(i \in \mathcal{N}, j \in \mathcal{T}_{i}, m \in \mathcal{M}_{i} \bigcap \mathcal{M}_{j} \text { and } \mathcal{I}_{q} \in \mathscr{I}\right) \\
& \sum_{q=1}^{|\mathscr{I}|} \lambda_{q} \leq 1, \lambda_{q} \geq 0 \quad\left(\mathcal{I}_{q} \in \mathscr{I}\right) \\
& f_{i j}(l) \geq 0\left(l \in \mathcal{L}, i \in \mathcal{N}, i \neq d_{t}(l), j \in \mathcal{T}_{i}, j \neq s_{r}(l)\right) \\
& \delta(l) \in\{0,1\} \quad(l \in \mathcal{L}),
\end{aligned}
$$

where $\delta(l), f_{i j}(l)$ and $\lambda_{q}$ are optimization variables, and $r(l)$ is deterministic value when session $l$ is given. Here, (11), (12) and (13) specify the routing constraints in spectrum clouds. (14) and (15) indicate that the flow rates over link $(i, j)$ cannot exceed the capacity of this CR link, which is obtained from 
the CR link scheduling as illustrated in Sec. III-B. Note that $\mathscr{I}$ includes all independent sets in CRNs. Given all the maximum 3-D independent $\operatorname{sets}^{7}$ in $\mathcal{G}(\mathcal{V}, \mathcal{E})$, we find that the formulated optimization is a mixed-integer linear programming (MILP) problem, which is NP-hard to solve as proved in [19], [28].

\section{The Upper Bound for the Cross-layer Optimization}

The complexity of the optimization above arises from two parts: (i) identifying all the maximum independent sets and (ii) fixing the binary $\delta(l)$ variables. To find all the maximum independent sets/cliques itself is NP-complete, but it is not a unique problem in spectrum clouds. It has been well investigated in prior multi-hop wireless networks and many approximation algorithms have been proposed in existing literature [17], [24], [26]. For example, one of the typical approaches is to employ $K(0 \leq K \leq|\mathscr{I}|)$ maximum independent sets (or a number of maximum conflict cliques) for approximation instead of finding out all the maximum independent sets in $\mathcal{G}(\mathcal{V}, \mathcal{E})$.

On the other hand, $\delta(l)$ variables will be involved as long as the SSP conducts the session based spectrum trading in multihop CRNs. Given all the maximum independent sets, we relax the binary requirement on $\delta(l)$ and replace it with $0 \leq \delta(l) \leq 1$ to reduce the complexity for the cross-layer optimization. Due to the enlarged optimization space (caused by relaxation on $\delta(l)$ ), the solution to this relaxed optimization problem yields an upper bound for the SSP's revenue maximization problem. Although the upper bound may not be achieved by a feasible solution, it can play as a benchmark to evaluate the quality of feasible solutions.

\section{A Bidding VAlue-Rate Requirement Ratio Based Heuristic Algorithm for Spectrum Trading}

In order to find feasible solutions, in this section, we propose a bidding value-rate requirement ratio $\left(\mathrm{BVR}^{3}\right)$ based heuristic algorithm for the SSP's revenue maximization problem. According to the bidding values and rate requirements of candidate trading sessions, we make the SSP classify those CR sessions into different categories in terms of decreasing access possibility. Then, we sequentially fix the $\delta(l)$-variables in different sets and give a heuristic solution, which is also a lower bound for the original MILP problem.

The key to simplifying the NP-hard optimization, fixing flow routing (i.e., $f_{i j}(l)$-variables) and link scheduling (i.e., $\lambda_{q}$-variables), and attaining a feasible solution is the determination of the binary values for the $\delta(l)$-variables [18], [20]. Although we can employ the classical branch-and-bound approach to determine $\delta(l)$-variables, the number of iterations involved in that algorithm grows exponentially with $|\mathcal{L}|$. To reduce the complexity, we propose a $\mathrm{BVR}^{3}$ based relax-and-fix algorithm [19]. The intuition behind the proposed algorithm is that given the leftover basic spectrum and the harvested spectrum, the SSP would like to take the best use of spectrum resources to make as much revenue as possible. That can be roughly interpreted as the SSP prefers to access the CR session with large bidding value and small rate requirements

\footnotetext{
${ }^{7}$ That is a general assumption used in existing literature [17], [23], [24] [26] for obtaining throughput bounds or performance comparison.
}

in spectrum clouds. The detailed procedure of the heuristic algorithm for the SSP's revenue maximization is presented as follows.

Based on bidding values and rate requirements of candidate CR sessions, we first sort all the CR sessions in terms of $\frac{b(l)}{r(l)}$ and partition these sessions into $S$ disjoint session sets $\mathcal{L}^{1}, \mathcal{L}^{2}, \cdots, \mathcal{L}^{S}$ in the order of decreasing $\mathrm{BVR}^{3}$, where $\bigcup_{s \in \mathcal{S}} \mathcal{L}^{s}=\mathcal{L}$ and $\mathcal{S}=\{1,2, \cdots, S\}$. The $\mathrm{BVR}^{3}$ of the session in $\mathcal{L}^{i}$ is larger than that of the session in $\mathcal{L}^{j}$, if $i$ is less than $j(\forall i, j \in \mathcal{S})$.

Then, we create auxiliary session sets by choosing subsets $\mathcal{A}^{s}$ with $\mathcal{A}^{s} \subseteq \bigcup_{u=s+1}^{S} \mathcal{L}^{u}$ for $s \in\{1,2, \cdots, S-1\}$. For example, in the spectrum trading problem, $\mathcal{L}^{1}$ may include the $\delta(l)$-variables associated with candidate trading sessions in $\left\{1,2, \cdots, l_{1}\right\}, \mathcal{L}^{2}$ may be associated with sessions in $\left\{l_{1}+1, l_{1}+2, \cdots, l_{2}\right\}$, and so on, whereas $\mathcal{A}^{1}$ would include the $\delta(l)$-variables associated with sessions in $\left\{l_{1}+1, l_{1}+\right.$ $\left.2, \cdots, a_{1}\right\}$, and so on.

By leveraging partitioned session sets (i.e., $\mathcal{L}^{s}$ ) and auxiliary session sets (i.e., $\mathcal{A}^{s}$ ), we sequentially solve $|\mathcal{S}|$ relaxedMILPs (R-MILPs) (denoted by $R$-MILP ${ }^{s}$ with $1 \leq s \leq|\mathcal{S}|$ ), determine the $\delta$-variables in $\mathcal{L}^{s}(s \in \mathcal{S})$ and find a heuristic solution to the original MILP problem. Specifically, in the first R-MILP, $R$-MILP ${ }^{1}$, we only impose the binary requirement on the $\delta(l)$-variables for session $l$ in $\mathcal{L}^{1} \cup \mathcal{A}^{1}$ and relax the integrality restriction on all the other $\delta(l)$-variables for session $l$ in $\mathcal{L}$. Thus, we have

$$
\begin{array}{lll}
R \text {-MILP } & \text { Maximize } & \sum_{l \in \mathcal{L}} b(l) \delta(l) \\
& & \\
\text { s.t.: } & (11),(12),(13), & (14),(15),(16) \\
& \delta(l) \in\{0,1\} & \left(\forall l \in \mathcal{L}^{1} \cup \mathcal{A}^{1}\right) \\
& \delta(l) \in[0,1] & \left(\forall l \in \mathcal{L} \backslash\left(\mathcal{L}^{1} \cup \mathcal{A}^{1}\right)\right)
\end{array}
$$

Let $\left\{\hat{\delta}^{1}(1), \cdots, \hat{\delta}^{1}(l), \cdots, \hat{\delta}^{1}(L)\right\}$ be an optimal solution to $R$-MILP $P^{1}$. We can fix the $\delta(l)$-variables in $\mathcal{L}^{1}$ at their corresponding binary values, i.e., $\delta(l)=\hat{\delta}^{1}(l) \in\{0,1\}$ for all $l \in \mathcal{L}^{1}$. Then, we move to $R-M I L P^{2}$.

In the subsequent $R-M I L P^{s}$ (for $2 \leq s \leq S$ ), we sequentially fix the binary values of the $\delta(l)$-variables for sessions in $\mathcal{L}^{s-1}$ from the solution to $R-M I L P^{s-1}$. After that, we further add the binary restriction for the $\delta(l)$-variables in $\mathcal{L}^{s} \cup \mathcal{A}^{s}$, and we have

$$
\begin{array}{cl}
R \text {-MILP } & \text { Maximize } \sum_{l \in \mathcal{L}} b(l) \delta(l) \\
\text { s.t.: } \quad(11),(12), & (13),(14),(15),(16) \\
\delta(l)=\hat{\delta}^{s-1}(l) & \left(\forall l \in \mathcal{L}^{1} \cup \cdots \cup \mathcal{L}^{s-1}\right) \\
\delta(l) \in\{0,1\} & \left(\forall l \in \mathcal{L}^{s} \cup \mathcal{A}^{s}\right) \\
\delta(l) \in[0,1] & \left(\forall l \in \mathcal{L} \backslash\left(\mathcal{L}^{1} \cup \cdots \cup \mathcal{L}^{s} \cup \mathcal{A}^{s}\right)\right) .
\end{array}
$$

Either $R-M I L P^{s}$ is infeasible for certain $s \in \mathcal{S}$ and the heuristic algorithm has failed, or else the proposed $\mathrm{BVR}^{3}$ based relax-and-fix algorithm provides a feasible solution (i.e., the solution to $R-M I L P^{|\mathcal{S}|}$ ) to the original MILP problem.

The core idea of the $\mathrm{BVR}^{3}$ based relax-and-fix algorithm is briefly summarized as follows. At each iteration, we solve a 
$R$-MILP $P^{s}$ problem involving $\mathcal{L}^{s} \cup \mathcal{A}^{s}$ sessions. To avoid being too myopic, we then only fix the $\delta(l)$-variables corresponding to sessions in $\mathcal{L}^{s}$. The auxiliary session sets $\mathcal{A}^{s}$ smooth the heuristic solution by creating some overlap between successive session sets. The procedure of the proposed heuristic algorithm is presented in Alg. 1.

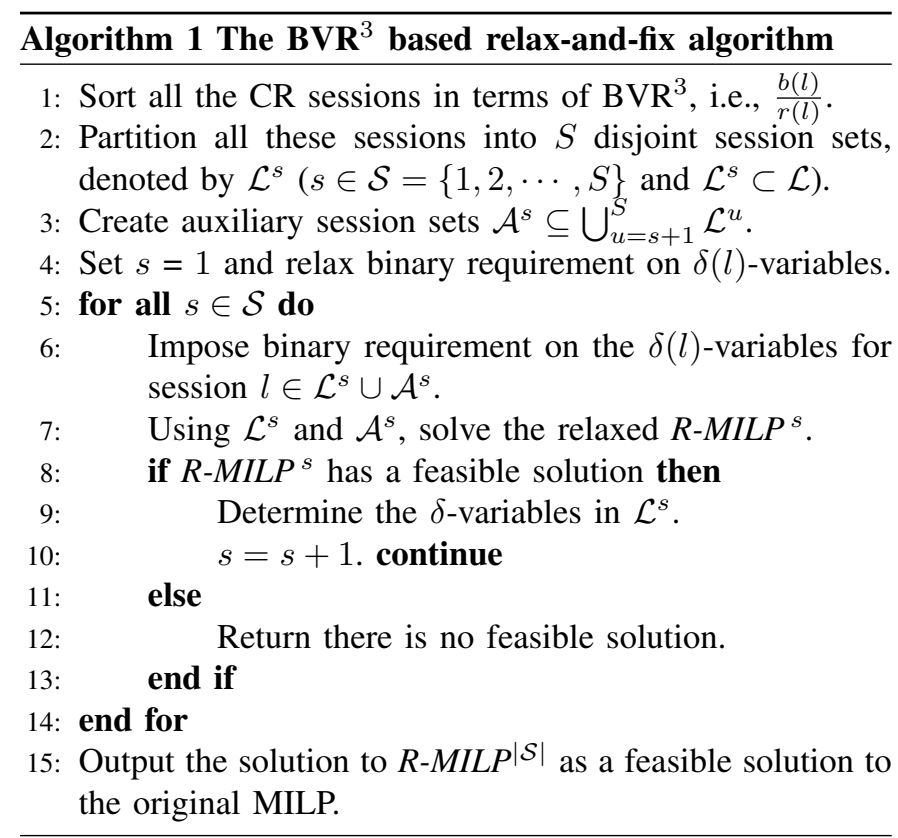

Different from the upper bound obtained in Sec. III-D, the proposed $\mathrm{BVR}^{3}$ based relax-and-fix algorithm yields a lower bound to the optimal spectrum trading problem formulated in Sec. III-C, provided that there exist feasible solutions.

\section{Performance Evaluation}

\section{A. Simulation Setup}

We consider a spectrum clouds in multi-hop CRNs consisting of a SSP, $|\mathcal{N}|=36 \mathrm{CR}$ mesh routers and $|\mathcal{L}|=18$ candidate trading sessions, each of which has a random rate requirement within $[10,30] \mathrm{Mb} / \mathrm{s}$. The bidding values of these sessions are within $[100,300]$. All CR mesh routers use the same power $P$ $=10 \mathrm{~W}$ for transmission. Considering the AWGN channel, we assume the noise power $\eta$ is $10^{-10} \mathrm{~W}$ at all routers. Moreover, suppose the path loss factor $\alpha=4$, the antenna parameter $\gamma$ $=3.90625$, the receiver sensitivity $P_{T}=100 \eta=10^{-8} \mathrm{~W}$ and the interference threshold $P_{T}=6.25 \times 10^{-10} \mathrm{~W}$. According to the illustration in Sec. II-C, we can calculate the transmission range $R_{T}$ and the interference range $R_{I}$, which are equal to $250 \mathrm{~m}$ and $500 \mathrm{~m}$, respectively. For illustrative purposes, we assume all the bands have identical bandwidth, which is set to be $10 \mathrm{MHz}$, i.e., $W^{m}=10 \mathrm{MHz}$ for all $m \in \mathcal{M}$. Besides, for the simplicity of computation, we set $K=3 \times 10^{4}$, i.e., if the total number of the maximum independent sets in $\mathcal{G}(\mathcal{V}, \mathcal{E})$ is less than or equal to $3 \times 10^{4}$, we employ all the maximum independent sets for the solution; otherwise, we employ $3 \times 10^{4}$ maximum independent sets for approximation.

Based on the simulation settings above, we conduct simulations to study the optimal spectrum trading problem in spectrum clouds with the following two topologies: i) a grid topology, where $36 \mathrm{CR}$ mesh routers are distributed within $1000 \times 1000 \mathrm{~m}^{2}$ area and the area is divided into 25 square cells in $200 \times 200 \mathrm{~m}^{2}$; ii) a random topology, where 36 CR mesh routers are randomly deployed in a $1000 \times 1000$ $\mathrm{m}^{2}$ area forming a connected network. Note that we employ CPLEX [29] to solve the relaxed optimization problems to obtain the upper bound and lower bound of the SSP's revenue.

\section{B. Results and Analysis}

In Fig. 3 and Fig. 4, we compare the upper bound of the SSP's revenue with the lower bound determined by the heuristic $\underline{B V R}^{3}$ based relax-and-fix algorithm (denoted by $\mathrm{BRF}$ in figures) at different number of available bands (i.e., $|\mathcal{M}|$ ) and radios (i.e., $|\mathcal{H}|$ ) in multi-hop CRNs. We relax the $\delta(l)$-variables and employ $K=3 \times 10^{4}$ maximum independent sets to solve the problem as illustrated in Sec. III-D, which also yields the upper bound. To develop the lower bound, we equally divide the 18 candidate trading sessions into 6 session sets (i.e., $|\mathcal{S}|=6$ and each set has 3 sessions) for the $\mathrm{BVR}^{3}$ based relax-and-fix algorithm as illustrated in Sec. IV. Given the number of available bands $|\mathcal{M}|$ in CRNs and radios $|\mathcal{H}|$ at CR routers, we employ 50 data sets that can produce feasible solutions and take the average value as a result. For each data set, we re-generate available bands $\mathcal{M}_{i}$ at $\mathrm{CR}$ router $i, s_{r}(l) / d_{t}(l)$ and $(r(l), b(l))$ pair of session $l$, and the random network topology (we keep the same grid topology for each data set), which follows the guideline of simulation setup.

From the results shown in Fig. 3 and Fig. 4, four observations can be made in order. First, the upper bound is close to the lower bound obtained from the proposed $\mathrm{BVR}^{3}$ based relax-and-fix algorithm, no matter how many available bands and radios are there in the spectrum clouds. We will further present the ratio of the upper bound to lower bound with 50 data sets in Fig. 5, analyze the statistical results and show the closeness between those bounds. Second, as the number of available bands and the number of CR mesh router's radios increase, the SSP's revenue increases as well. The reason is that more bands and radios available create more LBR tuples, so that more CR links in spectrum clouds may be activated for transmission simultaneously and more opportunities can be leveraged for spectrum trading in CRNs. However, the increment of the SSP's revenue basically stops when $|\mathcal{M}|$ is over 9 for $|\mathcal{H}|=2$ case in both grid topology and random topology, which leads to the third observation. That is, the CR mesh router has to equip a reasonable number of radios to utilize all the available bands efficiently (at least 3 radios for our simulation scenarios). This observation also gives a good suggestion on the design and deployment of CR mesh routers for spectrum clouds in practice. Fourth, the performance of the grid topology generally outperforms that of the random topology in terms of the SSP's revenue. The performance gap stems from the differences in topological structure. For the grid topology, each CR link has the same topological information if we ignore the border effect. The performance improvement of spectrum trading is mainly determined by the number of radios and the available bands at different CR routers. By contrast, the random topology is non-uniformed topology. The performance 

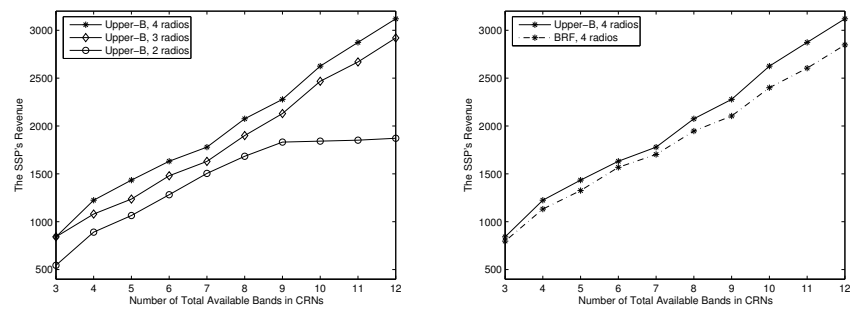

(a) Revenue upper bounds: $|\mathcal{H}|=2,3$ (b) Revenue upper bound and lower and 4 .

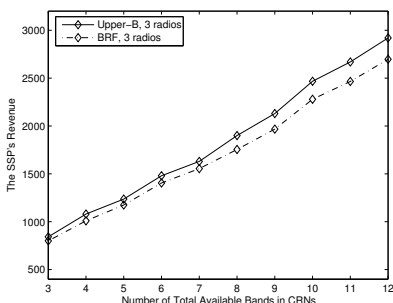

(c) Revenue upper bound and lower bounds: $|\mathcal{H}|=3$.

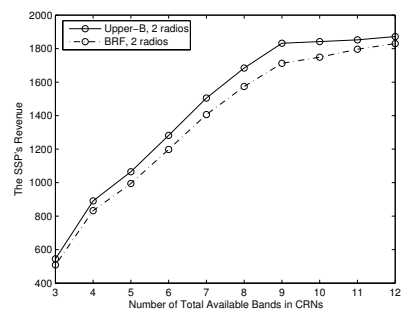

(d) Revenue upper bound and lower bounds: $|\mathcal{H}|=2$.

Fig. 3. Impact of the number of available bands $|\mathcal{M}|$ and radio interfaces $|\mathcal{H}|$ on spectrum trading in multi-hop CRNs: grid topology.
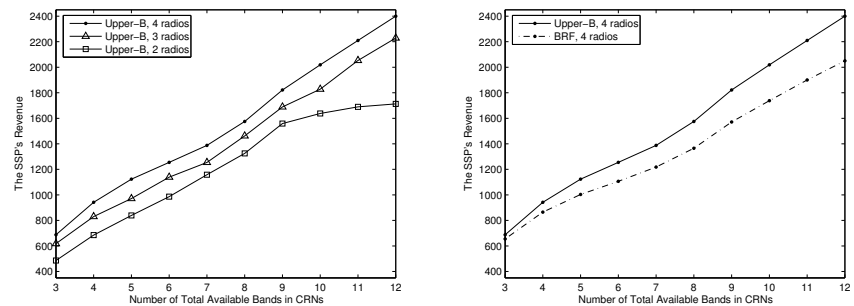

(a) Revenue upper bounds: $|\mathcal{H}|=2,3$ and 4 .

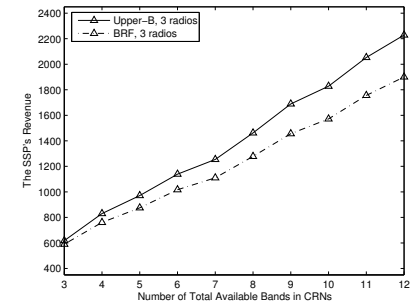

(c) Revenue upper bound and lower bounds: $|\mathcal{H}|=3$.

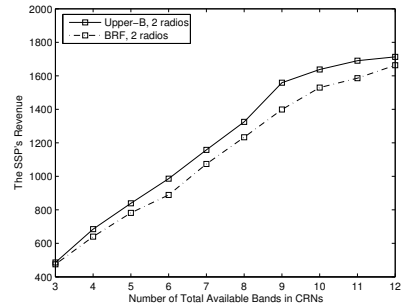

(d) Revenue upper bound and lower bounds: $|\mathcal{H}|=2$.

Fig. 4. Impact of the number of available bands $|\mathcal{M}|$ and radio interfaces $|\mathcal{H}|$ on spectrum trading in multi-hop CRNs: random topology.

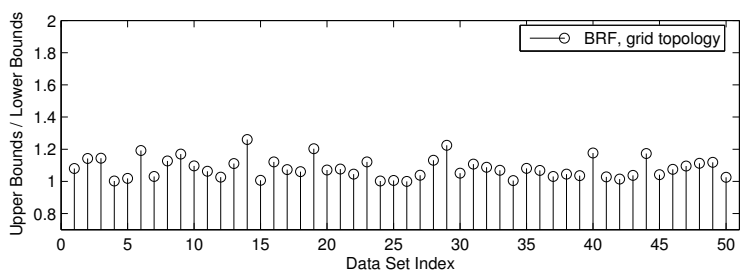

(a) Ratio of the upper bound to the lower bound determined by the $\mathrm{BVR}^{3}$ based relax-and-fix algorithm: grid topology.

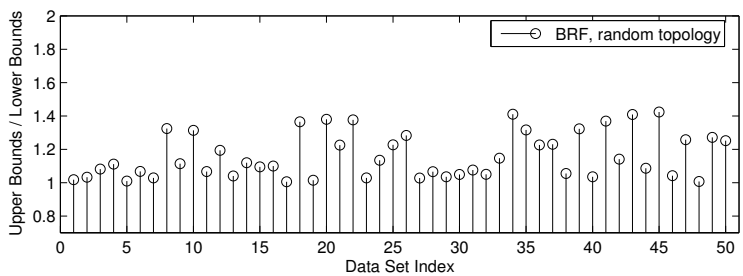

(b) Ratio of the upper bound to the lower bound determined by the BVR ${ }^{3}$ based relax-and-fix algorithm: random topology.

Fig. 5. Ratio of the upper bound to lower bounds determined by the proposed algorithms at $|\mathcal{H}|=3$ and $|\mathcal{M}|=9$.

improvement of spectrum trading is not only hindered by the number of bands and radios, but also bottlenecked by the critical cliques in the random topology.

Figure 5 presents the ratio of the upper bound to the lower bound obtained from the proposed heuristic algorithm in both grid topology and random topology, where $|\mathcal{H}|=3$ and $|\mathcal{M}|=$ 9. As shown in Fig. 5(a), the ratio of the upper bound to lower bound in the grid topology is near to 1 with 50 different data sets, where the lower bound is determined by the $\mathrm{BVR}^{3}$ based relax-and-fix algorithm. Specifically, the average ratio of the upper bound to the lower bound for all the data sets is 1.0826, and the standard deviation is 0.0632 . Similar analysis applies to the random topology as well. As shown in Fig. 5(b), the average ratio of the upper bound to the lower bound for all the data sets is 1.1611 , and the standard deviation is 0.1387 . All these statistical results indicate that the solutions found by the heuristic algorithm must be close to the optimum, since the optimal solution lies between the upper bound and the lower bound determined by the $\mathrm{BVR}^{3}$ based relax-and-fix algorithm.

Given the specific data set at $|\mathcal{H}|=3$ and $|\mathcal{M}|=9$, Table I(a) and Table $\mathrm{I}(\mathrm{b})$ present the trading status of the 18 candidate sessions w.r.t. $\mathrm{BVR}^{3}$ values in the grid topology and the random topology, respectively. The results demonstrate that unlike per-user based spectrum trading in CRNs, it is not necessary for the SSP to accommodate the CR sessions with high $\mathrm{BVR}^{3}$ values in order to maximize the SSP's revenue. Some other critical factors may also affect the results of the session based spectrum trading in multi-hop CRNs, e.g., the location of source/destination $\mathrm{CR}$ routers of a session, the interference a session incurs to the existing flows, etc. As shown in the formulation, the proposed spectrum clouds gives a comprehensive consideration on those factors. The data in Table I further verify this statement and explicitly show the advantages of our design over the per-user based spectrum trading systems in multi-hop CRNs.

\section{CONCLUSION}

In this paper, we have proposed a novel spectrum trading system, i.e., spectrum clouds, and presented a theoretical study on the optimal session based spectrum trading problem under multiple cross-layer constraints in multi-hop CRNs. We introduce a new service provider, SSP, and let the SSP provide coverage in CRNs with low-cost CR mesh routers in order to facilitate the accessing of SUs without CR capability. Considering the special features of session based spectrum trading, we exploit the 3-D (link-band-radio) conflict graph to 
TABLE I

SPECTRUM TRADING STATUS OF THE CANDIDATE SESSIONS W.R.T. THE DESCENDING BVR ${ }^{3}$ VALUES IN MULTI-HOP CRNS

(a) Grid topology with 3 radios and 9 bands

\begin{tabular}{|c|c|c||c|c|c|}
\hline S-Index & BVR $^{3}$ Val. & Status & S-Index & BVR $^{3}$ Val. & Status \\
\hline 1 & 24.171 & $\sqrt{ }$ & 10 & 15.629 & $\times$ \\
\hline 2 & 23.222 & $\sqrt{ }$ & 11 & 14.878 & $\sqrt{ }$ \\
\hline 3 & 22.411 & $\sqrt{ }$ & 12 & 14.627 & $\times$ \\
\hline 4 & 20.489 & $\sqrt{ }$ & 13 & 13.462 & $\times$ \\
\hline 5 & 20.014 & $\sqrt{ }$ & 14 & 12.239 & $\times$ \\
\hline 6 & 19.074 & $\sqrt{ }$ & 15 & 9.876 & $\sqrt{ }$ \\
\hline 7 & 18.475 & $\times$ & 16 & 8.358 & $\times$ \\
\hline 8 & 17.462 & $\sqrt{ }$ & 17 & 6.908 & $\times$ \\
\hline 9 & 16.081 & $\times$ & 18 & 4.912 & $\times$ \\
\hline
\end{tabular}

(b) Random topology with 3 radios and 9 bands

\begin{tabular}{|c|c|c||c|c|c|}
\hline S-Index & BVR $^{3}$ Val. & Status & S-Index & BVR $^{3}$ Val. & Status \\
\hline 1 & 23.188 & $\sqrt{ }$ & 10 & 11.672 & $\sqrt{ }$ \\
\hline 2 & 21.556 & $\sqrt{ }$ & 11 & 10.479 & $\times$ \\
\hline 3 & 20.139 & $\sqrt{ }$ & 12 & 9.002 & $\times$ \\
\hline 4 & 20.101 & $\sqrt{ }$ & 13 & 8.857 & $\times$ \\
\hline 5 & 18.525 & $\sqrt{ }$ & 14 & 6.971 & $\times$ \\
\hline 6 & 16.271 & $\times$ & 15 & 5.223 & $\times$ \\
\hline 7 & 14.771 & $\sqrt{ }$ & 16 & 4.713 & $\times$ \\
\hline 8 & 14.365 & $\times$ & 17 & 4.674 & $\times$ \\
\hline 9 & 13.213 & $\times$ & 18 & 3.737 & $\times$ \\
\hline
\end{tabular}

characterize the conflicts among CR links and mathematically describe the competitions among candidate trading sessions in spectrum clouds. Given the rate requirements and bidding values of candidate trading sessions, we formulate the optimal spectrum trading into the SSP's revenue maximization problem under the availability of spectrum, link scheduling and flow routing constraints in multi-hop CRNs. Since the formulated problem is NP-hard to solve, we derive an upper bound for the optimization by relaxing the integer variables. Furthermore, we propose heuristic algorithms for feasible solutions (low bounds as well). Through simulations, we show that: i) the proposed session based spectrum trading has superior advantages over the per-user based one in multi-hop CRNs; ii) the solutions attained by the proposed heuristic algorithms are near-optimal under different data sets in both the grid topology and the random one.

\section{REFERENCES}

[1] FCC, "Spectrum policy task force report," Report of Federal Communications Commission, Et docket No. 02-135, November 2002.

[2] J. Mitola, "Cognitive radio: An integrated agent architecture for software defined radio," Ph.D. Thesis, Royal Institute of Technology, Sweden, May 2000.

[3] I. Akyildiz, W. Lee, M. Vuran, and M. Shantidev, "Next generation/ dynamic spectrum access/ cognitive radio wireless networks: a survey," Computer Networks (Elsevier) Journal, vol. 50, no. 4, pp. 2127-2159, September 2006.

[4] D. Chen, S. Yin, Q. Zhang, M. Liu, and S. Li, "Mining spectrum usage data: a large-scale spectrum measurement study," in Proc. of international conference on Mobile computing and networking, ACM Mobicom, 2009, Beijing, China, September 2009.

[5] S. M. Mishra, D. Cabric, C. Chang, D. Willkomm, B. V. Schewick, A. Wolisz, and R. W. Brodersen, "A real time cognitive radio testbed for physical and link layer experiments," in Proc. of IEEE International Symposium on New Frontiers in Dynamic Spectrum Access Networks, DySPAN 2005, Baltimore, MD, November 2005.

[6] M. McHenry, "Spectrum white space measurements," New America Foundation Broadband Forum, June 20, 2003.
[7] M. A. McHenry, P. A. Tenhula, D. McCloskey, D. A. Roberson, and C. S. Hood, "Chicago spectrum occupancy measurements and analysis and a long-term studies proposal," in Proc. of TAPAS 2006, Boston, MA, August 2006.

[8] S. Sengupta and M. Chatterjee, "An economic framework for dynamic spectrum access and service pricing," IEEE/ACM Transactions on Networking, vol. 17, no. 4, pp. 1200-1213, Aug. 2009.

[9] X. Zhou, S. Gandhi, S. Suri, and H. Zheng, "ebay in the sky: strategyproof wireless spectrum auctions," in Proc. of Mobile Computing and Networking, Mobicom '08, San Francisco, CA, September 2008.

[10] X. Zhou and H. Zheng, "Trust: A general framework for truthful double spectrum auctions," in Proc. of INFOCOM 2009, Rio de Janeiro, Brazil, April 2009.

[11] J. Jia, Q. Zhang, Q. Zhang, and M. Liu, "Revenue generation for truthful spectrum auction in dynamic spectrum access," in Proc. of ACM International Symposium on Mobile Ad Hoc Networking and Computing, ACM MobiHoc, 2009, New Orleans, LA, May 2009.

[12] Y. Wu, B. Wang, K. J. Liu, and T. Clancy, "A multi-winner cognitive spectrum auction framework with collusion-resistant mechanisms," in Proc. of IEEE International Symposium on New Frontiers in Dynamic Spectrum Access Networks, DySPAN '08, Chicago, IL, October 2008.

[13] J. H. Reed, Software Radio: A Modern Approach to Radio Engineering. New York: Prentice Hall, May 2002.

[14] H. So, A. Tkachenko, and R. W. Brodersen, "A unified hardware/software runtime environment for FPGA based reconfigurable computers using borph," in Proc. of International Conference on Hardware-Software Codesign and System Synthesis, Seoul, Korea, October 2006.

[15] Defense Advanced Research Projects Agency (DARPA), "The neXt generation program (XG) official website." [Online]. Available: http://www.darpa.mil/sto/smallunitops/xg.html

[16] M. Pan, F. Chen, X. Yin, and Y. Fang, "Fair profit allocation in the spectrum auction using the shapley value," in Proc. of IEEE Global telecommunications conference, Globecom 2009, Honolulu, HI, USA, December 2009

[17] H. Li, Y. Cheng, C. Zhou, and P. Wan, "Multi-dimensional conflict graph based computing for optimal capacity in MR-MC wireless networks," in Proc. of International Conference on Distributed Computing Systems, ICDCS 2010, Genoa, Italy, June 2010.

[18] M. Pan, C. Zhang, P. Li, and Y. Fang, "Joint routing and scheduling for cognitive radio networks under uncertain spectrum supply," in Proc. of IEEE Conference on Computer Communications, INFOCOM 2011, Shanghai, China, April 2011.

[19] Y. Pochet and L. A. Wolsey, Production Planning by Mixed Integer Programming. Secaucus, NJ, USA: Springer-Verlag New York, Inc., 2006.

[20] Y. T. Hou, Y. Shi, and H. D. Sherali, "Spectrum sharing for multi-hop networking with cognitive radios," IEEE Journal on Selected Areas in Communications, vol. 26, no. 1, pp. 146-155, January 2008.

[21] A. Goldsmith, Wireless Communications. Cambridge, NY: Cambridge University Press, 2005.

[22] P. Gupta and P. R. Kumar, "The capacity of wireless networks," IEEE Transactions on Information Theory, vol. 46, no. 2, pp. 388-404, March 2000.

[23] J. Tang, S. Misra, and G. Xue, "Joint spectrum allocation and scheduling for fair spectrum sharing in cognitive radio wireless networks," Computer Networks (Elsevier) Journal, vol. 52, no. 11, pp. 2148-2158, August 2008.

[24] H. Zhai and Y. Fang, "Impact of routing metrics on path capacity in multirate and multihop wireless ad hoc networks," in Proc. of the IEEE International Conference on Network Protocols, ICNP 2006, Santa Barbara, CA, November 2006.

[25] Y. Shi, Y. T. Hou, and S. Kompella, "How to correctly use the protocol interference model for multi-hop wireless networks," in Proc. of ACM International Symposium on Mobile Ad Hoc Networking and Computing, ACM MobiHoc, 2009, New Orleans, LA, May 2009.

[26] F. Chen, H. Zhai, and Y. Fang, "Available bandwidth in multirate and multihop wireless ad hoc networks," IEEE Journal on Selected Areas in Communications, vol. 28, no. 3, pp. 299-307, April 2010.

[27] R. Diestel, Graph Theory. Springer, 2005.

[28] M. R. Garey and D. S. Johnson, Computers and Intractability: A Guide to the Theory of NP-Completeness. New York, NY: W. H. Freeman and Company, 1979.

[29] IBM ILOG CPLEX Optimizer. [Online]. Available: http://www-01.ibm. com/software/integration/optimization/cplex-optimizer/ 